\title{
Ipsilateral Breast Tumor Recurrence with Metachronous Contralateral Axillary Lymph Node Metastasis after Breast-Conserving Surgery with Axillary Lymph Node Dissection
}

\author{
Jin Gu Kang, M.D., Young Ah Kim, M.D., Jung Eun Choi, M.D., Ph.D., Soo Jung Lee, M.D., Ph.D., \\ Su Hwan Kang, M.D., Ph.D. \\ Department of Surgery, Yeungnam University College of Medicine, Daegu, Korea
}

\begin{abstract}
Contralateral axillary lymph node metastasis (CAM) is classified into synchronous and metachronous CAM. It is considered a stage IV disease by American Joint Community on Cancer (AJCC) cancer staging system. Although ipsilateral breast tumor recurrence (IBTR) with metachronous CAM is rare, it can occur after previous axillary lymph node dissection (ALND) because of altered lymphatic drainage. Metachronous CAM might be a regional disease progression rather than a distant metastasis. Here, we present a case of IBTR with metachronous CAM. This patient was treated with curative intent. The management of CAM remains controversial.
\end{abstract}

Key Words: Breast neoplasms, Lymph nodes, Neoplasm metastasis, Recurrence

\section{INTRODUCTION}

The ipsilateral axillary lymph node is usually the lymphatic drainage site in breast cancer [1]. Metachronous contralateral axillary lymph node metastasis (CAM) is defined as an axillary lymph node recurrence after primary treatment of breast cancer. Some studies have reported that breast cancer can spread to the contralateral axillary lymph node by permeating the deep lymphatic channel after a sentinel lymph node biopsy (SLNB) or an axillary lymph node dissection (ALND). Therefore, CAM could occur because of an altered lymphatic drainage pathway after ALND [2,3]. However, CAM is uncommon, with a prevalence ranging from $1.9 \%$ to $6 \%$. It is usually considered a distant metastasis [4]. One study reported that metachronous CAM patients who underwent ALND, axillary radiation, chemotherapy, and endocrine therapy had an overall survival rate of $82.6 \%$ after a mean follow-up period of 50.3 months [5]. Another study showed a longer overall disease-free survival time of metachronous CAM patients (without other metastatic diseases) than synchronous CAM patients [6]. Ipsilateral breast tumor recurrence (IBTR) with CAM after ALND is uncommon, its manage-

Correspondence: Su Hwan Kang, M.D., Ph.D.

Department of Surgery, Yeungnam University College of Medicine, 170 Hyunchoong-ro, Namgu, Daegu 42415, Korea

Tel: +82-53-620-3580, Fax: +82-53-624-1213, E-mail: kangsuhwan@yu.ac.kr

Received: Aug 3, 2019 Revised: Nov 28, 2019 Accepted: Oct 21, 2020 ment remains controversial. We report a case of IBTR with metachronous CAM after ALND. This study was approved by the Institutional Review Board of Yeungnam University College of Medicine (IRB No. 2019-06-035).

\section{CASE REPORT}

A 29-year-old woman visited our center last year for further evaluation of a left breast skin lesion. She was married for two years and was nulliparous. She underwent a left breast-conserving surgery with left ALND after being diagnosed with invasive breast cancer 4 years ago in her country. At that time, the final pathological result was invasive ductal carcinoma (IDC), pT1N0M0, estrogen receptor (ER) or progesterone receptor (PR) positive, and human epidermal growth factor receptor 2 (HER2) negative. She received adjuvant chemotherapy, doxorubicin and cyclophosphamide (AC), followed by docetaxel, radiotherapy, and endocrine therapy (Tamoxifen $20 \mathrm{mg}$ daily). She had a reddish skin lesion on the lower inner quadrant of the left breast 3 months ago. On physical examination, segmentally distributed reddish skin lesions and strawberry-like nipple coloration were found on her left breast (Figure 1). First, a punch biopsy for the skin lesion and incisional biopsy of the nipple were performed, along with mammography (MMG) and ultrasonography (USG). There were multiple small 


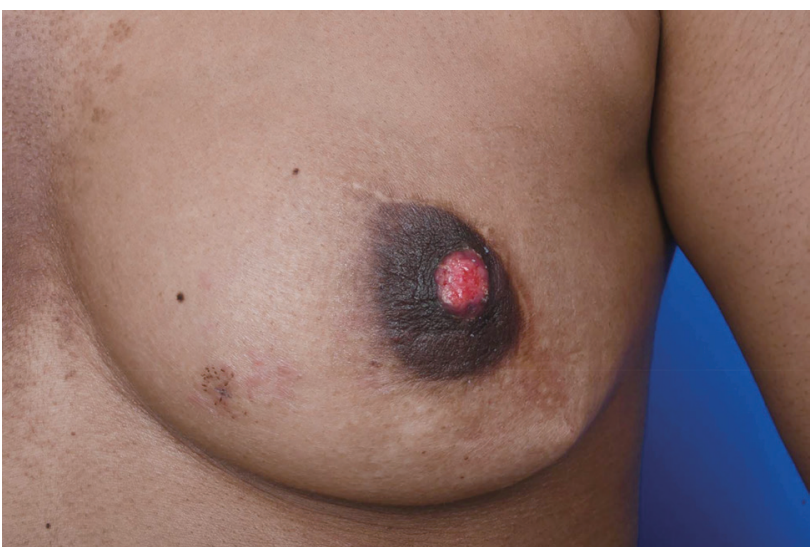

Figure 1. Strawberry-like nipple and segmentally distributed reddish skin lesion on the lower inner quadrant of the left breast.

hypoechoic nodules in the left subareolar area, detected on ultrasonography (Figure 2A). She then underwent ${ }^{18} \mathrm{~F}$-fluorodeoxyglucose positron emission tomography/computed tomography (FDG PET-CT) and magnetic resonance imaging (MRI). MRI showed left nipple and peri-areolar skin thickening with a non-mass enhancement extending $4 \mathrm{~cm}$ (Figure 2B). PET-CT showed an unusual finding of a contralateral axillary lymph node with focal FDG uptake (Figure 2C). MRI and PET-CT showed no evidence of malignant lesions in the right breast. Preoperative incisional biopsy revealed Paget's disease with invasive carcinoma of the left nipple. She underwent fine-needle aspiration for the right axillary suspicious lymph node, which showed metastasis. Paget's disease with invasive carcinoma for the left nipple with skin and right axillary lymph node metastasis was diagnosed preoperatively based on pathologic results. Before surgery, we recommended counseling by a gynecologist for cryopreservation. However, they were against cryopreservation and genetic examination, such as that for BRCA 1/2. We performed a left salvage mastectomy with a right ALND and left breast reconstruction using a latissimus dorsi flap. The final pathological diagnosis of the left breast revealed IDC with Paget's disease, lymphatic tumor emboli, measuring $1.3 \times 0.6 \mathrm{~cm}$, ER positive, PR negative, HER2 negative, and Ki-67 30\% (Figure 3A, 3B). Right ALND revealed 2/9 lymph node metastases without extranodal extension (Figure 3C). Metastatic lymph nodes were ER positive, PR positive, HER2 negative, and Ki-67 positive (25\%-30\%). She underwent chemotherapy (four cycles of AC followed by four cycles of docetaxel every three weeks). Adriamycin was administered at a total cumulative dose of $440 \mathrm{mg} / \mathrm{m}^{2}$, considering possible cardiac toxicity. Radio-
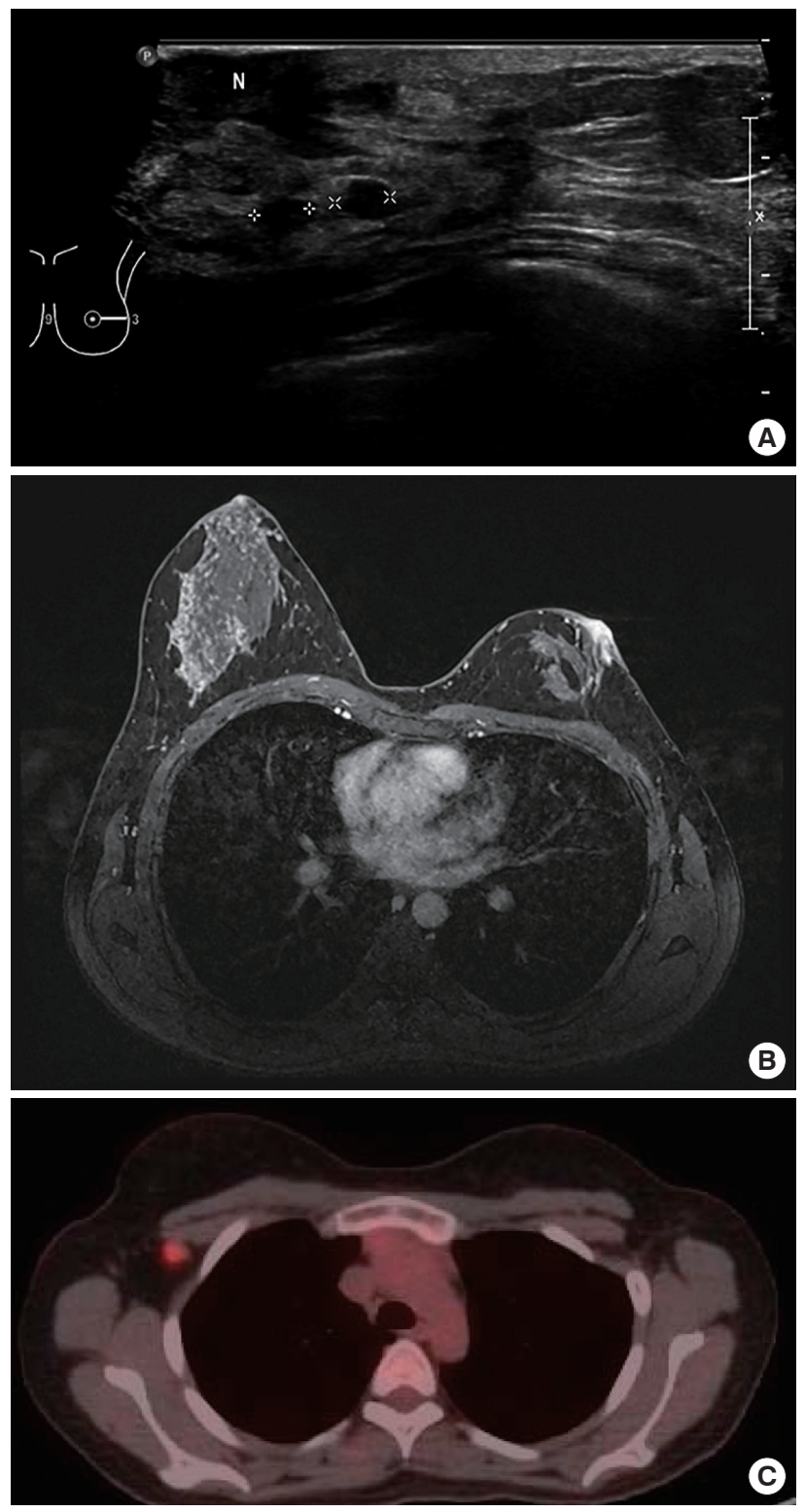

Figure 2. Ipsilateral breast tumor and contralateral axillary lymph node as preoperative radiologic findings. (A) Ultrasonography showing multiple hypoechoic lesions in the left subareolar area. (B) Magnetic resonance imaging showing $4 \mathrm{~cm}$ extent non-mass enhancement at the left subareolar area with peri-areolar skin enhancement. (C) ${ }^{18} \mathrm{~F}$-fluorodeoxyglucose positron emission tomography/computed tomography showing contralateral axillary metastasis (maximum standardized uptake value $=3.81$ ) without any other metastasis.

therapy (RT) was performed for the whole right breast, right axilla, internal mammary area, supraclavicular, and infraclavicular areas. Endocrine therapy (Tamoxifen $20 \mathrm{mg}$ daily) was initiated as well as ovarian function suppression. Follow-up imaging included MMG, USG, MRI, abdominal and chest CT, and bone scan. There was no evidence 

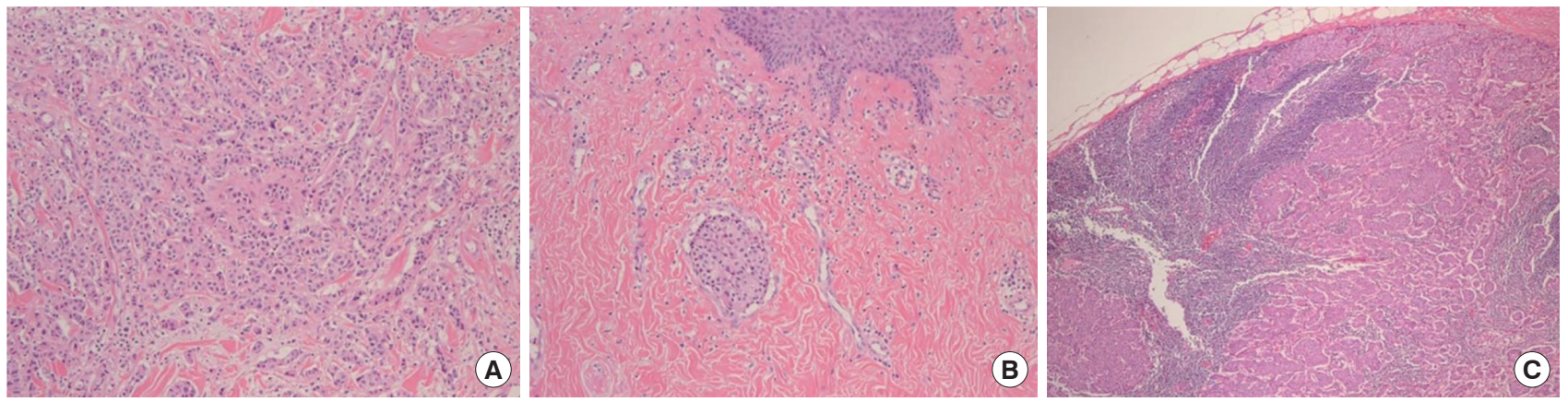

Figure 3. Postoperative microscopic findings showing ipsilateral breast tumor recurrence and contralateral axillary lymph node metastasis. (A) Left breast specimen showing infiltrating ductal carcinoma (hematoxylin and eosin $[\mathrm{H} \& \mathrm{E}], \times 100$ ). (B) Lower inner quadrant of the left breast skin revealing lymphatic tumor emboli and the space around the tumor nest lined by endothelium $(H \& E, \times 100)$. (C) Right axillary lymph node specimen revealing metastatic infiltrating ductal carcinoma $(\mathrm{H} \& \mathrm{E}, \times 40)$.

of recurrence of cancer 15 months post-treatment.

\section{DISCUSSION}

IBTR after breast-conserving surgery (BCS) was divided into true recurrence and new primary disease. True recurrence (TR) occurs when the residual cancer grows gradually, making it hard to detect. It is characterized by a high rate of lymph node (LN) metastasis (37.8\%) and a short disease-free interval (mean: 46.6 months). A new primary (NP) is defined as de novo cancer occurrence, independently. It exhibits a low level of lymph node metastasis (8.7\%) and a longer disease-free interval (mean: 62.1 months). The five-year survival rate after IBTR was $71.0 \%$ in TR and $94.7 \%$ in NP patients ( $p=0.022$ ). Ten-year overall survival rates of TR and NP patients were $71.2 \%$ and $92.3 \%$, respectively. NP showed a significantly better survival rate than TR $(p=0.02)$ [7]. IBTR with CAM in the absence of distant metastasis is rare. CAM can occur owing to aberrant lymphatic flow. A disruption in the ipsilateral lymphatic channel can result in redirection of lymph flow to other nodal basins such as the contralateral internal mammary and contralateral axillary nodes. Drainage to the contralateral axilla, in the absence of drainage to the ipsilateral axilla, is significantly higher in patients with a previous axillary dissection than in patients with SLNB $[6,8,9]$. CAM has been reported in a few patients with various prognoses. Although the American Joint Committee on Cancer staging classifies $\mathrm{CAM}$ as a distant disease, its staging remains controversial. One study reported that CAM is associated with aggressive tumor characteristics (large tumors, grade 3, lymphovascular invasion), and a high incidence of HER2 positive tumors [10]. CAM patients have a poor prognosis, with a relapse rate of $>70 \%$ and a mortality rate of 25\% within 2-3 years [4]. Moossdorff et al. [5] reviewed 24 articles to evaluate outcomes of CAM patients without other distant metastatic diseases. In their systemic review, 48 patients were divided into three groups (26: isolated CAM, 7: IBTR with clinically CAM, and 15: IBTR with CAM). The survival rate was $76.9 \%$ in patients with isolated CAM and $83.4 \%$ in patients with IBTR and CAM. This prognosis is similar to that of regional recurrence [5]. Based on these studies, metachronous CAM might be a regional event rather than a distant metastasis $[5,6,8,9]$. In a systemic review of 24 articles, ALND for 21 patients (70.8\%), chemotherapy for 21 patients (43.8\%), endocrine therapy for 7 patients (14.6\%), and regional radiotherapy for 3 patients (6.3\%) were administered [5]. Chemotherapy for isolated locoregional recurrence (ILRR) of breast cancer (CALOR) trial showed that the overall survival and disease-free survival for patients with ER-negative ILRR increased significantly with chemotherapy. Adjuvant chemotherapy should be recommended for patients with ER-negative ILRR [11]. Hormonal therapy is the first treatment choice with an excellent response for hormone receptor-positive tumors [10].

Our case report showed that IBTR with metachronous CAM could occur after BCS with ALND because of altered lymphatic drainage. However, its management remains controversial. If there is no evidence of distant metastasis, it might be necessary to treat IBTR and metachronous CAM with curative intent.

\section{CONFLICT OF INTEREST}

The authors declare that they have no competing interests. 


\section{REFERENCES}

1. Nieweg OE. Lymphatics of the breast and the rationale for different injection techniques. Ann Surg Oncol 2001;8:71S-73S.

2. Tokmak H, Kaban K, Muslumanoglu M, Demirel M, Aktan S. Management of sentinel node re-mapping in patients who have second or recurrent breast cancer and had previous axillary procedures. World J Surg Oncol 2014;12:205.

3. Maaskant-Braat AJ, Voogd AC, Roumen RM, Nieuwenhuijzen GA. Repeat sentinel node biopsy in patients with locally recurrent breast cancer: a systematic review and meta-analysis of the literature. Breast Cancer Res Treat 2013;138:13-20.

4. Wang W, Yuan P, Wang J, Ma F, Zhang P, Li Q, et al. Management of contralateral axillary lymph node metastasis from breast cancer: a clinical dilemma. Tumori 2014;100:600-4.

5. Moossdorff M, Vugts G, Maaskant-Braat AJ, Strobbe LJ, Voogd AC, Smidt ML, et al. Contralateral lymph node recurrence in breast cancer: regional event rather than distant metastatic disease. A systematic review of the literature. Eur J Surg Oncol 2015;41:1128-36.

6. Chkheidze R, Sanders MAG, Haley B, Leitch AM, Sahoo S. Isolated contralateral axillary lymph node involvement in breast cancer represents a locally advanced disease not distant metastases. Clin Breast Cancer 2018;18:298-304.

7. Komoike Y, Akiyama F, Iino Y, Ikeda T, Tanaka-Akashi S, Ohsumi S, et al. Analysis of ipsilateral breast tumor recurrences after breastconserving treatment based on the classification of true recurrences and new primary tumors. Breast Cancer 2005;12:104-11.

8. Barranger E, Montravers F, Kerrou K, Marpeau O, Raileanu I, Antoine $\mathrm{M}$, et al. Contralateral axillary sentinel lymph node drainage in breast cancer: a case report. J Surg Oncol 2004;86:167-9.

9. Lizarraga IM, Sugg SL, Weigel RJ, Scott-Conner CE. Review of risk factors for the development of contralateral breast cancer. Am J Surg 2013;206:704-8.

10. Morcos B, Jaradat I, El-Ghanem M. Characteristics of and therapeutic options for contralateral axillary lymph node metastasis in breast cancer. Eur J Surg Oncol 2011;37:418-21.

11. Aebi S, Gelber S, Anderson SJ, Lang I, Robidoux A, Martin M, et al. Chemotherapy for isolated locoregional recurrence of breast cancer (CALOR): a randomised trial. Lancet Oncol 2014;15:156-63. 